\title{
Analytical-Numerical Modeling Approach for Calculation of the Structural Distortions after Welding and Thermal Straightening
}

\author{
Vesselin G. Michailov and Nikolay Doynov \\ Department Joining and Welding Technology, Brandenburg University of Technology, Cottbus 03046, Germany
}

\begin{abstract}
The analytic-numerical hybrid model for calculating welding distortions in large welded structures is presented. Objective of the analytical model is the calculation of the plastic strains and their distribution after welding and thermal straightening process. The consideration of the essential physical relations is put into discussion. Afterwards the obtained plastic strains by the analytical calculation are loaded on an elastic FE-model of the structure and the distortions of the whole structure are predicted. The consideration of welding and thermal straightening scenarios and the assembling stages is done by taking into account the intermediate variation of the strain state at every processing step. The model is intended to be used for solving industrial tasks, i.e. intending acceptable precision and calculation time as well as low simulation costs. The application of the model is demonstrated on structures with many welds and straightening spots.
\end{abstract}

Key words: Computational welding mechanics, welding distortions, thermal straightening.

\section{Introduction}

The welding distortions are still one of the main problems in welding technology. They decrease the quality of the welded structure and complicate the smooth accomplishment of the manufacturing process. Since several decades thermal straightening is an established technique for improving welding structures. By the thermal straightening additional deformations are put into the structure, which lead to decreasing the out-of-plane distortion and increasing the in-plane shrinkage. Prediction and control of the welding distortions and the thermal straightening process, during the early stages of a design cycle, allow avoiding expensive engineering corrections.

Nowadays the welding as well as the straightening process guiding and parameters are chosen on the background of practical experience, complemented with additional time- and cost-intensive experiments. Unfortunately this approach does not allow capturing

Corresponding author: Nikolay Doynov, Ph.D., IWE, res. ass., research field: computational welding mechanics. the influence of the different structural stiffness and residual stresses state at every manufacturing stage.

Another approach seems to be a welding-thermal straightening distortion simulation, based on the computational welding mechanics. Approaches to the problem, particularly in welding, are covered by analytical or numerical models. The application of analytical models is strongly limited due to the necessary simplifications of the physical phenomena. Today, the most common simulation procedure is the coupled thermo-metallurgical-mechanical FE- simulation, which allows very accurate prediction of welding distortions. The industrial application of these welding simulations, enlarged with straightening simulations, is not feasible because of the long computation time and costs. These difficulties rise when the subject of the simulation is a large, complex welded structure and often makes it inefficient. Hence, there is a great demand for simple welding simulation models and approaches which allow for quick distortions prediction.

The aim of this work is to create an approach for solving the structural displacement during the entire 
welding - thermal straightening cycle with particular emphasis on the industrial application.

\section{Modeling Approach}

An innovative solution approach for simulation of welding distortions has been developed by Michailov et al. [1, 2]. The approach is an integration of analytical and numerical calculation's procedures in a hybrid model. The analytical procedure calculates the shrinkage volume i.e. strains distribution in the weld seams region, based on the theoretical works of Okerblom [3], Gatovskij [4] and Vinokurov [5]. The calculated strains are then applied to the global elastic FE model (Fig. 1) of the structure to predict the distortions during welding as well as their residual state. The change of the stress state after each weld as well as of the structure stiffness after each assembly step is used as initial condition for analytical calculation of the plastic strain (shrinkage volume) of the next one.

In this work a conception for an analytical model for thermal straightening as well as its coupling with the welding hybrid model is realized. The creation of a new comprehensive engineering tool on is achievable under the condition that the models with the appropriated simplifications will deliver results with acceptable precision. Therefore, the model must have strong theoretical base and its application limitations should be clearly defined. In addition all relevant influence factors should be quantified by means of mathematical expressions.

\section{Analytical Model}

Purpose of the analytical model is the determination of the plastic strain and the zone where it appears, as shrinkage volume. The mechanical background of the model for welding is based on the kinetic of the plastic strains under temperature dependent ideal elastic-plastic material behavior [5-7]. In case of long welds the mechanical problem can be considered as uniaxial stress-strain problem with normal perpendicular to the

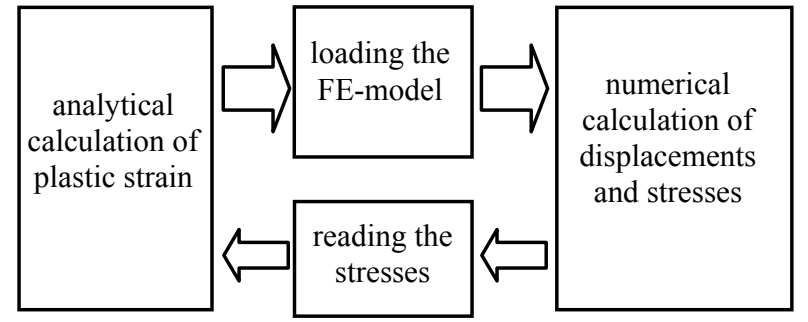

Fig. 1 Analytical-numerical hybrid model for welding and thermal straightening.

weld cross section (beams theory) and with known distribution of the maximal temperatures in it. The thermal strain (expansion and shrinkage) in the longitudinal direction is constraint by the surrounding cold metal, which realizes infinity restrain. According to this model the zone with the plastic strains $b_{p z}$ is defined by certain maximal temperatures, i.e. by the maximal thermal strain $\alpha\left(\Delta T_{\max }\right)$ equal to yield strain $\varepsilon_{Y}$ and $2 \varepsilon_{Y}$, Fig. 2a. The introduction of complex semi-empirical relations allows to take into consideration the relevant factors and to reach the need adequacy.

The longitudinal weld shrinkage mainly depends on the occurring maximum temperature increment $\Delta T_{\max }$ while welding. In case of thin wall structures, for instance, it can be solved by modification of Rykalin's [8] partial solutions for the maximum temperatures by high speed heat source $[2,9]$ :

$$
\Delta T_{\max }(y)=\frac{q_{s}}{\delta \rho c v} \frac{\sqrt{\chi}}{\sqrt{\pi \sqrt{4 \chi y^{2}+1}-1}} e^{-\frac{\sqrt{4 x y^{2}+1}}{2}}
$$

where, $q_{s}$ is the heat, $\delta$, the plate thickness, $c$, the specific heat, $\rho$, the density, $v$ travel speed, and $\chi$ is the quotient of $b$, the heat exchange number and $a$, the temperature transfer coefficient. Thus, for maximal value of the thermal strain $\varepsilon_{m}^{\text {th }}$ we obtain:

$$
\varepsilon_{m}^{t h}(y)=\alpha \Delta T_{\max }(y)
$$

where, $\alpha$ is the expansion coefficient. Note that the entities $\alpha, \rho$, and $c$ are temperature dependent but their quotient is near constant in the essential range from room temperature till $600{ }^{\circ} \mathrm{C}[5,9]$. 


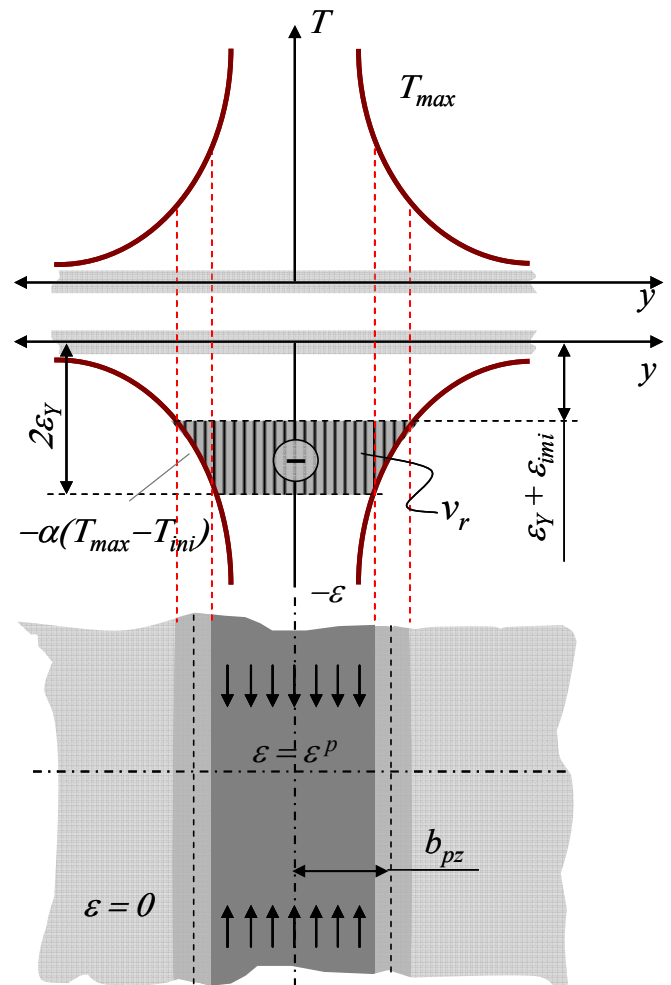

(a) transverse distribution of the longitudinal plastic strains in a weld seam

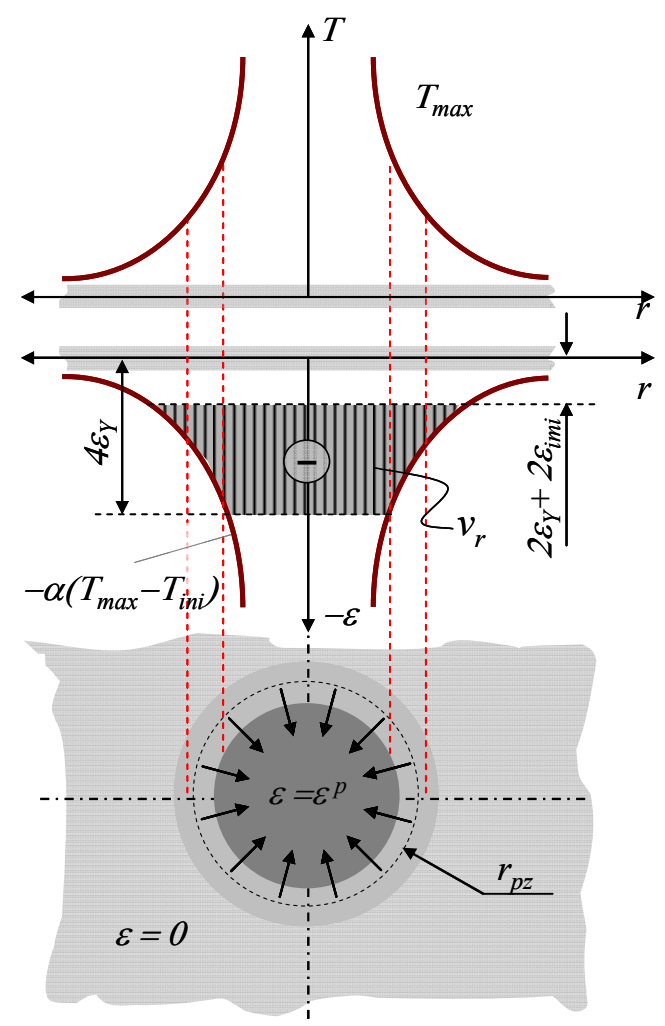

(b) radial distribution of the plastic strains in a heat spot

Fig. 2 Distribution of plastic strain.
If separate Eq. (1) from the coefficient, considering the heat exchange with the environment in thin plates (introducing $K_{\alpha}$ ) we receive rather simple expression, which, written in respect to coordinate $y$ and integrated between $\varepsilon_{Y}$ and $2 \varepsilon_{Y}$ gives:

$$
v_{x}=\int_{\varepsilon_{Y}}^{2 \varepsilon_{Y}} \sqrt{\frac{2}{\pi e}} \frac{\alpha}{c \rho} \frac{q_{s}}{\varepsilon_{T}} d \varepsilon K_{\alpha}=0.335 \frac{\alpha}{c \rho} q_{s} K_{\alpha}
$$

If the heated zone is pre-stressed, i.e. is imposed either to external loads or to residual stresses from previous welds, the width of plastic zone should be determined after addition of the initial strains $\left(\varepsilon_{i n i}\right)$ to the thermal one. Thus, the lower limit of the integration interval becomes $\varepsilon_{Y}+\varepsilon_{i n i}$. Since the simplification with introduction of $K_{\alpha}$ is made a direct integration is not allowed and the dependence on the initial strain is taken into account by means of separate function $K_{\sigma}$. In that way, the welding sequence or changes by the clamping condition and structural restrain could be taken into account. Further, introducing different functions, additional effects on the plastic strain, such as relation of the heat input to the thickness $K_{\alpha S}$, external and internal stiffness $K_{k}$, solid state transformations $K_{u}$ etc. can be taken into consideration [6]. The integral value of transverse plastic strains is calculated in similar way, taking into account the variation of the restrain in the heated domain due to the relative elongation of the temperature field in longitudinal direction [6].

Hence, the longitudinal and transversal shrinkage volume $v_{x}$ and $v_{y}$, follow from Refs. [2, 6]:

$$
\begin{gathered}
v_{x}=0.335 \frac{\alpha}{c \rho} q_{s} K_{\alpha} K_{\alpha s} K_{k} K_{u} K_{\sigma} \\
v_{y}=\frac{\alpha}{\rho c} q_{s}\left(1+K_{\mu} K_{s}\right)\left[1+K_{c}\left(1-K_{s}\right)\right] \times \\
\times\left[0.255+0.745 K_{s}\left(0.04+0.96 K_{a v} K_{v}\right)\right] \times \\
\times K_{a b} K_{\sigma}+\frac{\varepsilon_{s}}{A_{p r}}\left(1-K_{s}\right)
\end{gathered}
$$

Capturing all these effects on the arising distortions simultaneously is not possible as they affect 
each other. For this reason their determination is done iteratively $[2,6]$.

If the heat source has circular shape, as it occurs by heat point straightening, the kinetics of the strain development varies. Assuming a large circular disk, imposed on heating at its center and followed by cooling, an axial symmetric mechanical problem should be considered. In that case the surrounding cold metal behaves elastically either by heating or by cooling and the arising strain in it is equal to the elastic strain in the heated core. Hence, in a non pre-stressed disc the plastic strains increase from zero to its maximal value in domain between maximal thermal strain $\alpha \Delta T_{\max }(r)$ equal to yield strain $2 \varepsilon_{Y}$ and $4 \varepsilon_{Y}$ respectively, $r_{p z}$ zone on Fig. $2 b$. Presence of any initial strain, assuming homogeneity in the heat point, will shift the lower limit of integration interval with two times the initial value, i.e. it becomes $2 \varepsilon_{Y}+$ $2 \varepsilon_{i n i}$.

To apply that theoretical model an appropriate solution of the thermal problem is need. In common case, the solutions with instantaneous heat source $[8,9]$ give satisfactory results. Considering, for instance, instantaneous line heat source on a thin plate [8] the distribution of maximal temperature increment becomes:

$$
\Delta T_{\max }(r)=\frac{Q_{1}}{\rho c \pi r^{2}} e^{-1-\beta r^{2}}
$$

where, $Q_{1}$ is the effective heat per length and $\beta$ denotes the heat exchange number. This equation, in analogy to solution for weld seam, can be simplified by means of $K_{\alpha}$, i.e. correction function considering the cooling condition. Thus, writing the relation of thermal strain and maximal temperature distribution, in analogy to Eq.

(1), in respect to the radius we get:

$$
r\left(\varepsilon_{m}^{t h}\right)=\sqrt{\frac{\alpha Q_{1}}{\rho c \pi e \varepsilon_{m}^{t h}}} K_{\alpha}=\sqrt{\frac{A}{\varepsilon_{m}^{t h}}} K_{\alpha}
$$

Further, the integration of Eq. (2) in the interval $2 \varepsilon_{Y}$ and $4 \varepsilon_{Y}$ gives the shrinkage volume for a plate without initial stress. Extending the integration interval, in order to take into account the influence of the initial strain can be done by introducing an additional correction function $K_{\sigma}$. Finally, the shrinkage volume becomes:

$$
v_{r}=\int_{2 \varepsilon_{Y}}^{4 \varepsilon_{Y}} r\left(\varepsilon_{m}^{t h}\right) d \varepsilon=2(2-\sqrt{2}) \sqrt{A \varepsilon_{s}} K_{\alpha} K_{\sigma}
$$

Both $K_{\alpha}$ and $K_{\sigma}$ functions differ from these, used for welding seam calculation. For instance, their particular solutions for heat point can be expressed by means of dimension less parameter $A_{\beta}$, as derived in (Fig. 3): $A_{\beta}=\frac{\alpha Q_{1}}{\rho c \pi e} \frac{\beta}{\varepsilon_{Y}}$, as well as the normalized strain: $\hat{\varepsilon}_{i n i}=\frac{\varepsilon_{i n i}}{\varepsilon_{Y}}=\frac{\sigma_{i n i}}{\sigma_{Y}}$.
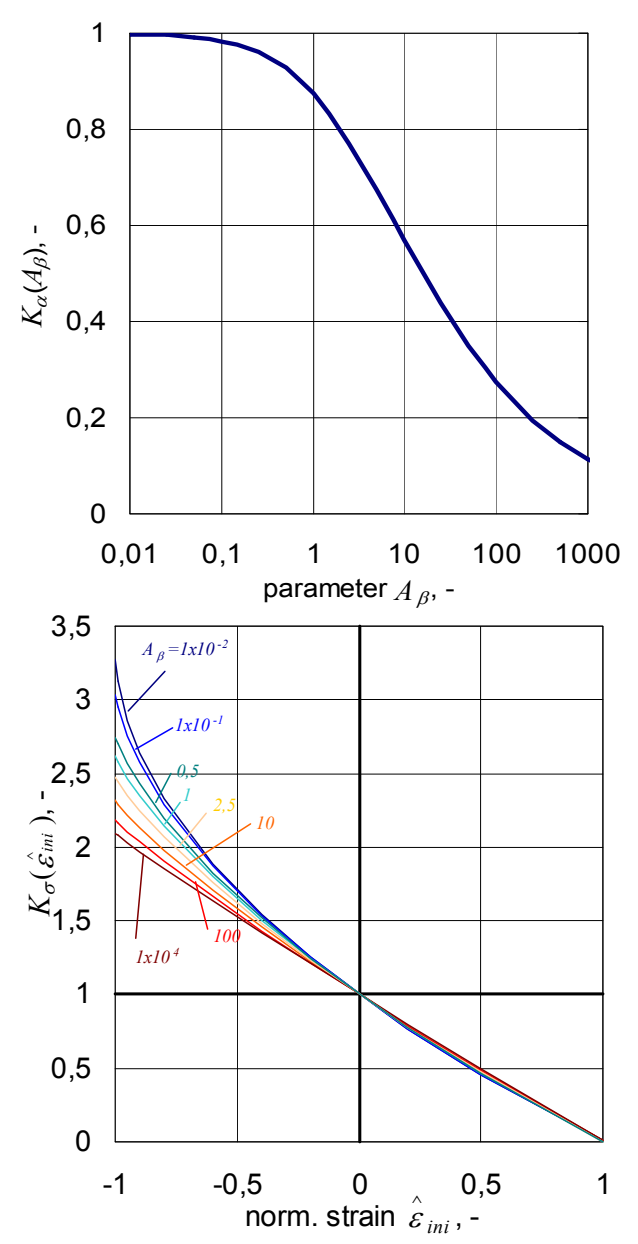

Fig. 3 Solutions of influence functions $K_{\alpha}$ and $K_{\sigma}$ for various $A_{\beta}$ and normalized initial strain. 
To apply that model the initial strain in the treated domain must be known, i.e. an appropriate solution of the structural response is need. In common case, such solution can be obtained by means of numerical simulation $[1,2]$.

\section{Numerical Model}

In order to predict the distortions as well as the stresses in the entire structure the calculated shrinkages are applied as local loads to the FE-model and an simple elastic analysis, subsequentially after each weld or straightening point is carried out. In this way the stresses in the domain of the next weld or heat point can be obtained, by means of a post-processing routine and put to the analytical solution as initial state i.e. solving $K_{\sigma}$. In current case an averaging of the elastic strain $\varepsilon^{e}$ on the top, middle or bottom plane (index $z$ ) of the elements $j=1$...m assigned to the area with dimensions $\delta_{x} \times \delta_{y}$ (the area subject to loading) is used:

$$
\hat{\varepsilon}_{i n i, i, z}=\frac{1}{\delta_{x} \delta_{y}} \sum_{j=1 \ldots m} S_{e_{j}} \varepsilon_{i, z j}^{e}
$$

where, $S_{e}$ is the element surface.

As the numerical solution encloses the structural stiffness as well as the displacements and stresses from the previous loads it is possible to take into consideration the influence of the welding sequence, changes of the clamping conditions and structure assembly.

There are different possibilities for loading the finite element model of the structure. The most self-evident technique is the loading by shrinkage strain, which is given by:

$$
\varepsilon=\frac{v_{i}}{A_{p l}},
$$

where $v_{i}$ denotes the shrinkage volume in longitudinal $\left(v_{x}\right)$, transverse $\left(v_{y}\right)$ or radial $\left(v_{r}\right)$ direction respectively and $A_{p l}$ the area of the plastic zone, i.e. the area bounded by $b_{p z}$ or $r_{p z}$. In addition the strain can be distributed over the plate thickness by the help of the centroid of the plastic zone $z_{c}[1,2,6]$ :

$$
\varepsilon(z)=\varepsilon+\frac{12 \varepsilon z_{c}}{s^{2}} z
$$

where, $z$ is the coordinate of the integration point along the shell thickness.

In common case a shell finite element model with full integration $(2 \times 2)$ and with 3 or 5 integration points along the thickness is used. The element size in the domains, subject to loads can be as large as the plastic zone is. Since the solution is linear elastic, only the general material constants, i.e. Young's module and Poasson's coefficient are needed.

\section{Application of the Hybrid Model}

To demonstrate the capability of the hybrid model a welding simulation, followed by simulation of a thermal straightening was carried out. The scope is a qualitative demonstration, i.e. plausibility proof of the model. A complex structure with 16 welds and dimensions $500 \times 400 \mathrm{~mm}$ was chosen in Fig. 4 . It is made from four long-bars joined with the plate by intermittent laser beam welds 1 and 2, two crossbars joined with the plate by intermittent laser beam welds 3 and 4 and with the long-bars by MSG welds 5 till 16 (Fig. 4a). Wall thickness of the plate and the bars is 2 $\mathrm{mm}$. The material is a Cr-Ni $18-10$ structural steel. All bars ware preliminary fixed by means of spot welds, however, it was neglected by the calculation. Further, reference straightening parameters considering a real production process ware chosen. The welding sequence follows the weld numbers.

Object of analysis is the final in plane distortions of the structure, i.e. the final dimensions in the plane after welding and straightening. For application of the hybrid model a finite element model with rectangular shell elements was generated, using ANSYS $^{\circledR}$ FE-Code. The mesh is very coarse and just refined in the areas imposed to loading (marked areas in Fig. 4b). Thus, in those domains the element size along the welds is about $20 \mathrm{~mm}$. The loading is realized by 


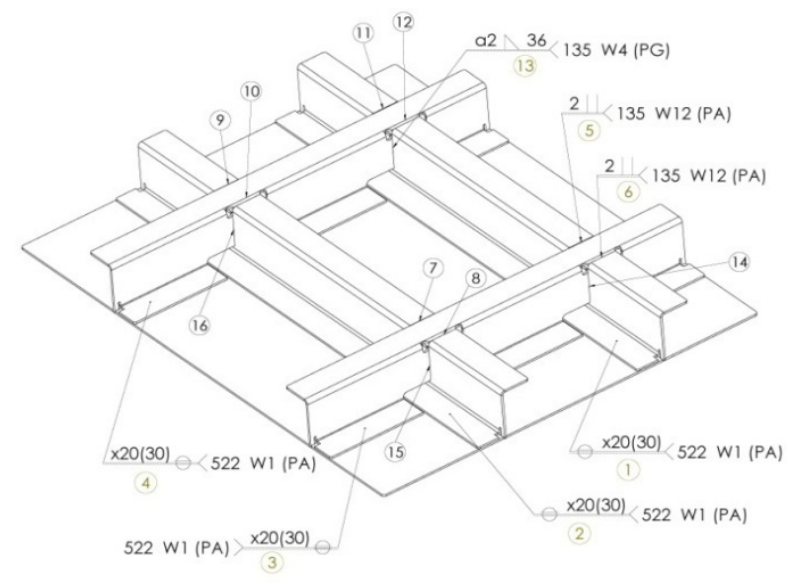

(a) drawing with weld symbols (upside down)

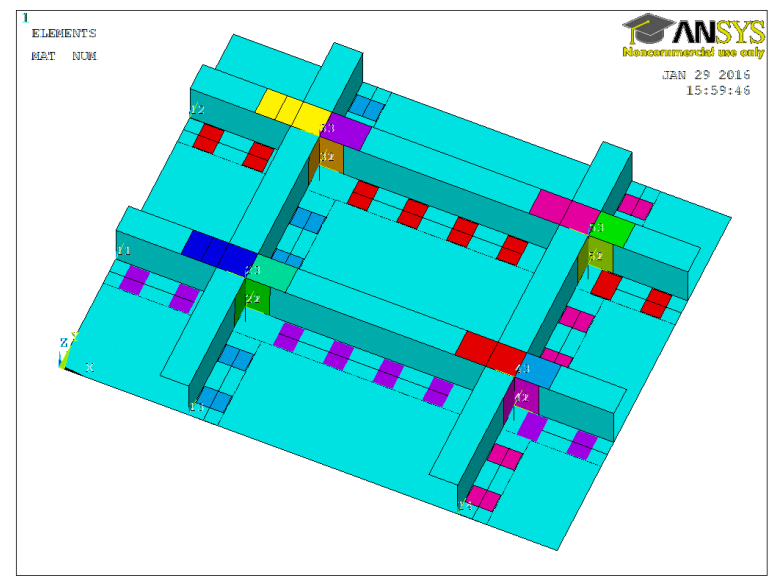

(b) solid model, with the domains subject of loading during welding (upside down)

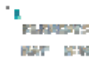

ONN IS

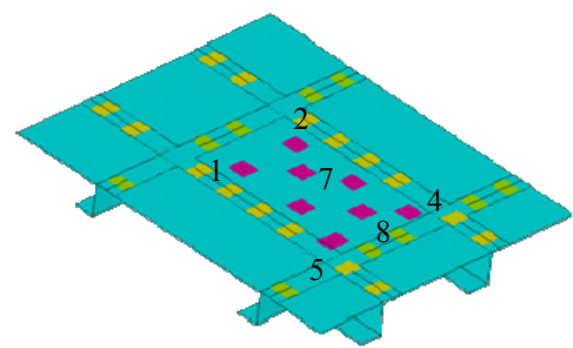

(c) solid model, with the domains subject of loading during thermal straightening

\section{Fig. 4 Welded structure.}

longitudinal and transverse strains (on element coordinate system), linear distributed along the thickness, in sixteen load steps, following the welding sequence, see marked areas in Fig. 4b.

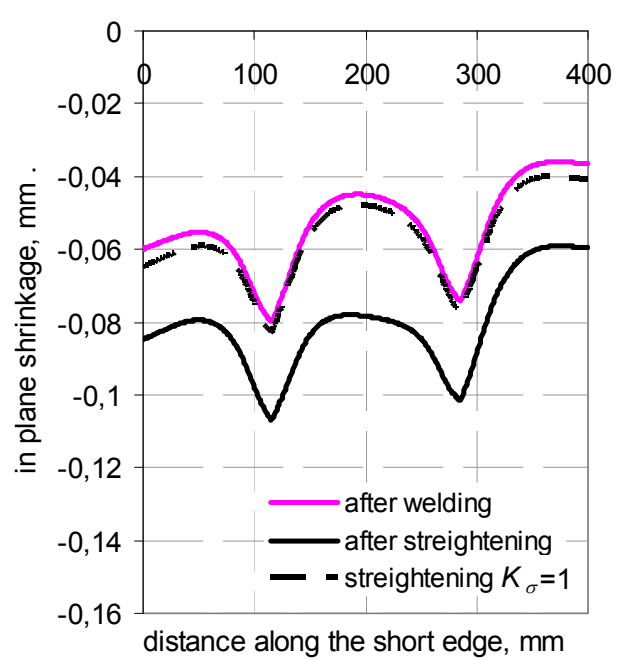

(a) longitudinal shrinkage

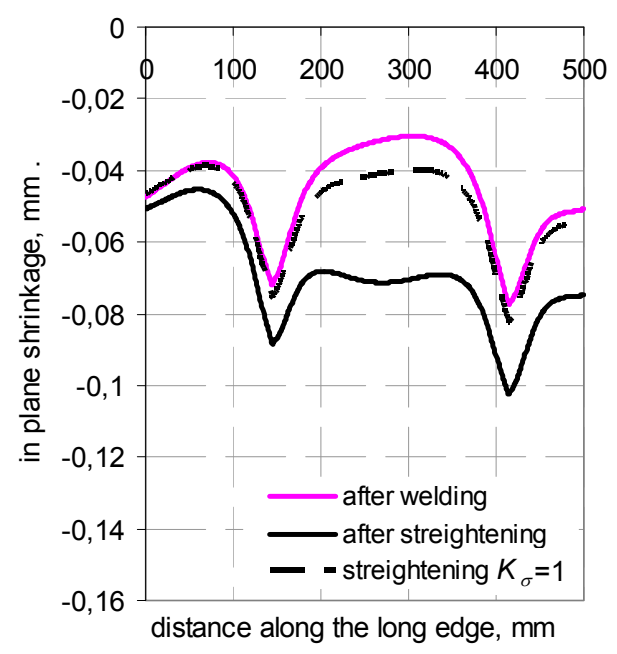

(b) transverse shrinkage

Fig. 5 Comparison of calculated in-plane displacement of the structure after welding and after thermal straightening, scenario with 8 heat points.

The strains as well as their centroid are calculated by means of the analytical model, stated above, taking into consideration the stress variation after every one welds. The red curves in Fig. 5 show the obtained shrinkage in longitudinal (a) and transverse (b) direction, i.e. the difference between initial and final (after welding) structural dimensions.

Based on the calculated welding distortions and stresses a thermal straightening simulation was carried out. It was done by means various scenarios with 2 to 15 heat spots, where the nominal width of plastic zone corresponds to about $18 \mathrm{~mm}$. The heating pattern is 
drawn in spiral, setting the points consistently from outside toward the centre of the plate. The plastic strain for every next spot was calculated, taking into consideration the actual elastic strain vector from the current elements. At every stage the calculation was done according to the relation $K_{\sigma}$, stated above and thus the width of the plastic zone varied from point to point. The calculation shows an increasing of in-plane shrinkage, exemplary in case of 8 points straightening scenario, with about 20\% (black curves in Fig. 5). Additionally, a calculation the same scenario without taking into consideration of the stress variation during straightening, i.e. keeping alloys $K_{\sigma}=1$, shows severe deviation from the correct solution (dashed curves in Fig. 5). The examination of intermediate steps as well as the final state shows that the variations in the plastic zone width during the process are reasonable. Preliminary measurements have validated the plausibility of calculation.

The calculation time for the entire simulation, welding and straightening, took 10-20 min. The obtained results, after the last spot, confirm the expected result-accumulating additional in-plane shrinkage. Hence, the consideration of stress variation is required.

To validate the model a comparison with 3-D optical measurements was used. The calculated results are presented exemplary by the field of displacement in Z-direction (out-of-plane), and compared with measurements, Fig. 6 . The distortions, obtained by the hybrid model satisfy the experimental data. Both, measured (Fig. 6a) and calculated (Fig. 6b) fields, show angular distortions, which produce out of plane displacements toward the profile in the vicinity of the weld seems about $1.7 \mathrm{~mm}$ ( $\mathrm{Z}$ negative direction). In the same time the bending of the sheet edges is in Z-positive direction, opposite to the frame, and reaches a value of about $2.4 \mathrm{~mm}$. Sleight visual disagreements can be explained with the scattering of the measured signals. In this study the maximal error, measured as absolute deviation related to the dimensions of the

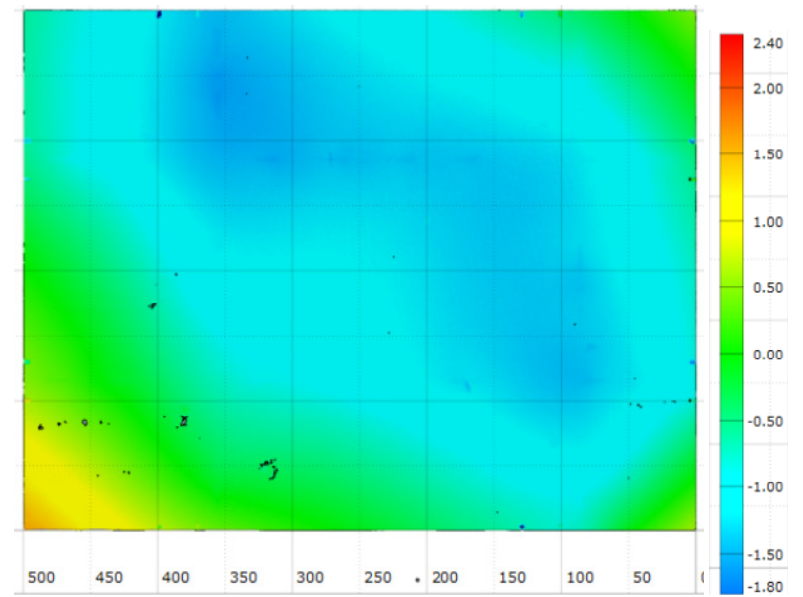

(a) measured displacement in $\mathrm{mm}$

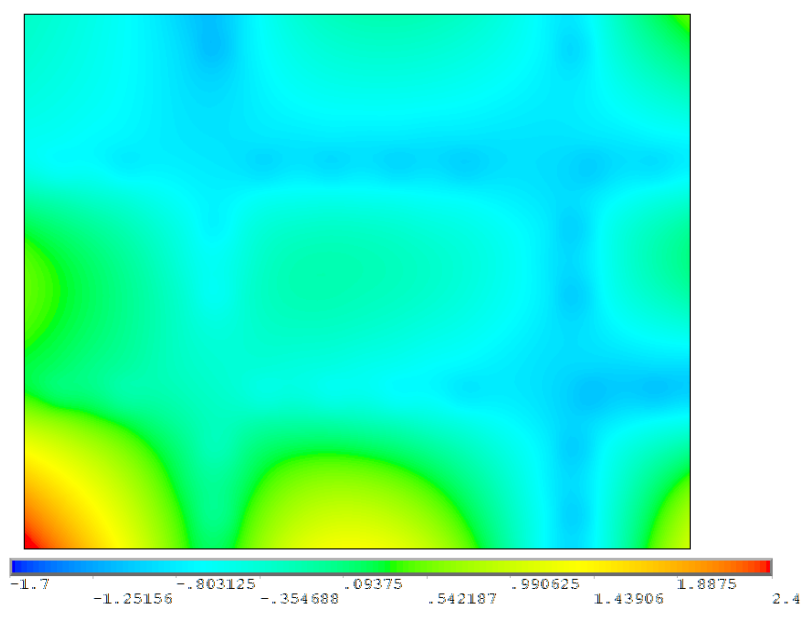

(b) calculated displacement in $\mathrm{mm}$

Fig. 6 Comparison of the field of out of plane displacements in the sheet of the structure after welding.

structure is lower than $8 \%$. Thus, the middle uncertainty of the simulation approach in the current case can be assessed as $0.25 \%$, which is rather lower than the common manufacturing tolerances. So, the model is absolutely feasible for solving industrial tasks.

\section{Conclusions}

The work gives overview of a hybrid analytical numerical simulation approach for solving the distortion problem in welding production, dedicated to welding and straightening procedures. The suggested approach overcomes the limitation of common thermo-mechanical simulation and allows employment of computational methods in the welding production, 
with special emphasis on large welded structures. The presented analytical-numerical approach has strong theoretical base. The main advantage of the approach is the ability to take into account the major influence factors and especially the stress variation during the manufacturing process. In this way it is possible to consider exactly the sequence of welding and straightening works and thus to achieve an acceptable precision. In the same time it is simple to apply. It needs simple input, temperature independent material constants and general process parameters, i.e. accessible or easy to determine data, as intended to meet the needs for solving industrial tasks.

To demonstrate the model capability an analysis of welding distortions, after welding and subsequent thermal straightening in a large structure with 16 welds and 8 straightening points has been carried out. Furthermore the qualitative interaction of the thermal straightening and the welding processes is analyzed for a reference scenario. For this purpose the variation of the stress state in the structure, during welding and straightening is taken into consideration. The results of the analytic-numerical hybrid model match the expertise and the expectations. Additional calculation was carried out in order to qualify and quantify the error if the stress variation, i.e. residual stresses after welding, is not taken into consideration. The last approves the general concept of the model.

The comparison of the calculated displacements with measurements for a certain experimental test validates the hybrid analytical numerical model. The achieved uncertainness is comparable with the manufacturing tolerances. Thus the potential for application in the industry is assured.

The calculation time was about several minutes on common PC. The coupled analytic-numerical model shows enormous potential regarding to the reduction of the computation time, as well as significant reduction of the pre-processing time.

\section{Acknowledgement}

This work was carried out within the research project IGF-Nr: 17970 BR of the "FOSTA e.V." in the framework of the AiF-program to promote the "Industrielle Gemeinschaftsforschung und -entwicklung (IGF)" and financed by the German Federal Ministry of Economics and Technology (BMWi) on the basis of a decision by the German Bundestag.

\section{References}

[1] Michailov, V. G., Doynov, N., Stapelfeld, C., and Ossenbrink, R. 2011. "Hybrid Model for Prediction of Welding Distortions in Large Structures." Front. Mater. Sci. 5 (2): 209-15.

[2] Stapelfeld, C. 2016. Vereinfachte Modelle zur Schweißverzugsberechnung, Dissertation, Berichte des Lehrstuhls Füge- und Schweißtechnik der BTU Cottbus-Senftenberg, Band 10.

[3] Okerblom N. O. 1955. Welding Stresses in Metal Structures, Mashinostroenie, Moskau/Leningrad. (in Russian)

[4] Gatovskii, K. M., and Karkhin, V. A. 1980. Theory of Welding Deformations and Stresses, LKI, Leningrad, 1980 (in Russian).

[5] Vinokurov, V. A. 1968. Welding Stresses and Distortion, Mashinostroenie, Moskau. (in Russian)

[6] Kuzminov, S. A. 1974. Welding Deformations of Ship-Structures, Sudostroenie, Leningrad. (in Russian)

[7] Ueda, Y., Fukuda, K., and Kim, Y. C. 1978. "Restraint Stresses and Strains due to Silt Weld in Rectangular Plates." Trans. LWRI 7 (1): 11-6.

[8] Rykalin, N. 1957. Berechnung der Wärmevorgänge beim Schweißen, VEB Verl. Technik, Berlin.

[9] Michailov, V. G., Karkhin, V. A., and Petrov, P. P. 2012. Fundametals of Welding, Strojtelni Konstrukcii OOD. (in Bulgarian) 